\title{
Modeling genetic and nongenetic variation of feed efficiency and its partial relationships between component traits as a function of management and environmental factors
}

\author{
Y. Lu, ${ }^{*}$ M. J. Vandehaar, ${ }^{\star}$ D. M. Spurlock,† K. A. Weigel,‡ L. E. Armentano,‡ C. R. Staples,§ E. E. Connor,\# \\ Z. Wang,II M. Coffey, , R. F. Veerkamp, ${ }^{* *}$ Y. de Haas, ${ }^{* *}$ and R. J. Tempelman ${ }^{* 1}$ \\ *Department of Animal Science, Michigan State University, East Lansing 48824 \\ †Department of Animal Science, lowa State University, Ames 50011 \\ ‡Department of Dairy Science, University of Wisconsin, Madison 53706 \\ §Department of Animal Sciences, University of Florida, Gainesville 32611 \\ \#Animal Genomics and Improvement Laboratory, Agricultural Research Service, USDA, Beltsville, MD 20705 \\ IIDepartment of Agricultural, Food and Nutritional Science, University of Alberta, Edmonton, AB, T6G 2P5 Canada \\ IAnimal and Veterinary Sciences Group, Scottish Agricultural College (SAC), Roslin Institute Building, Easter Bush, Midlothian, EH25 9RG, \\ United Kingdom \\ ${ }^{* *}$ Animal Breeding and Genomics Centre, Wageningen UR Livestock Research, $6700 \mathrm{AH}$ Wageningen, the Netherlands
}

\section{ABSTRACT}

Feed efficiency (FE), characterized as the fraction of feed nutrients converted into salable milk or meat, is of increasing economic importance in the dairy industry. We conjecture that FE is a complex trait whose variation and relationships or partial efficiencies $(\mathrm{PE})$ involving the conversion of dry matter intake to milk energy and metabolic body weight may be highly heterogeneous across environments or management scenarios. In this study, a hierarchical Bayesian multivariate mixed model was proposed to jointly infer upon such heterogeneity at both genetic and nongenetic levels on $\mathrm{PE}$ and variance components (VC). The heterogeneity was modeled by embedding mixed effects specifications on $\mathrm{PE}$ and $\mathrm{VC}$ in addition to those directly specified on the component traits. We validated the model by simulation and applied it to a joint analysis of a dairy FE consortium data set with 5,088 Holstein cows from 13 research stations in Canada, the Netherlands, the United Kingdom, and the United States. Although no differences were detected among research stations for $\mathrm{PE}$ at the genetic level, some evidence was found of heterogeneity in residual PE. Furthermore, substantial heterogeneity in $\mathrm{VC}$ across stations, parities, and ration was observed with heritability estimates of $\mathrm{FE}$ ranging from 0.16 to 0.46 across stations.

Key words: dry matter intake, genetic correlation, heritability, hierarchical Bayesian modeling

Received May 19, 2016.

Accepted September 1, 2016.

${ }^{1}$ Corresponding author: tempelma@msu.edu

\section{INTRODUCTION}

Feed efficiency $(\mathbf{F E})$ is becoming more important for the economic and environmental sustainability of dairy production and increases as a greater proportion of feed nutrients are directed toward milk production (Connor, 2015). A commonly used measure of $\mathrm{FE}$ is residual feed intake (RFI), which is defined as the difference between actual and predicted DMI. That is, RFI responses are typically derived as the estimated residuals from a linear model analysis whereby partial regression relationships are specified between DMI and energy sink covariates such as milk energy (MILKE) and metabolic BW (MBW), for example. Given that there has been some reluctance to directly incorporate RFI in breeding goals for several different reasons (Berry and Pryce, 2014), some investigators have focused their attention on DMI as the key phenotype for $\mathrm{FE}$ analyses (Berry et al., 2014; de Haas et al., 2015).

Recently, Lu et al. (2015) proposed a multiple-trait (MT) mixed model analysis of DMI with MILKE and MBW that further resolves whether RFI or DMI should be considered as the key response variable for FE. They demonstrated that Cholesky decompositions performed on each of the estimated (e.g., by REML) $3 \times 3$ genetic and residual variance-covariance matrices among the 3 key traits (MILKE, MBW, and DMI) lead to a parameterization whereby the estimated partial regression relationships between DMI with MILKE, and between DMI with MBW are essentially partitioned into genetic and residual components. Additionally, the Cholesky decomposition leads to a determination of EBV for $\mathrm{FE}$ that is identical to that based on a classical RFI analysis under the special case whereby the partial regression 
relationships relating DMI to MILKE and to MBW are specified to be identical at both genetic and residual levels. As a corollary, Lu et al. (2015) demonstrated by simulation that the greater the discrepancy between the partial relationships at genetic and nongenetic levels, the greater the EBV accuracy for their proposed MT approach relative to a classical RFI analysis. Furthermore, the MT approach facilitates the incorporation of data on cows that might be missing, even selectively, on any of the 3 key phenotypes that would otherwise be discarded in a RFI analysis (Pollak et al., 1984).

A hierarchical Bayesian extension of a 2-trait MT model was developed earlier in a nongenetic context by (Bello et al., 2010) who later inferred heterogeneous partial regression relationships between calving intervals and milk yield at both herd and cow levels as a function of environmental and herd management factors for Michigan dairy herds (Bello et al., 2012). We surmise that the genetic and residual partial relationships between DMI with MILKE, or between DMI with MBW, could also be modeled as a linked multifactorial function of various factors including parities, research stations, and rations, for example. Indeed, based on station-specific RFI analyses, Tempelman et al. (2015) determined that estimated partial regression coefficients of DMI on MILKE and on MBW were highly heterogeneous across research stations in several countries. Furthermore, adaptation of the hierarchical Bayesian approach as proposed by Bello et al. (2010) would also infer the degree of heterogeneity in heritabilities of $\mathrm{FE}$ across different management conditions.

The 2 primary objectives of this study were (1) to identify potential management or environmental factors that might affect genetic and residual partial regression relationships (i.e., partial efficiencies; PE) between DMI with MILKE, and between DMI with MBW, and (2) to determine whether there is evidence of heterogeneity of genetic and residual variances for $\mathrm{FE}$ across these same management or environmental factors.

\section{MATERIALS AND METHODS}

\section{Multiple Trait Model}

Our developments closely combine developments provided in $\mathrm{Lu}$ et al. (2015) with those provided in Bello et al. (2010). The 3 key component traits of FE are numbered as follows in the MT model: (1) MILKE, (2) MBW, and (3) DMI. We write this MT model as follows:

$$
\mathbf{y}_{i}=\mathbf{X}_{i} \boldsymbol{\beta}+\mathbf{Z}_{i} \mathbf{u}+\mathbf{e}_{i} .
$$

Here, $\mathbf{y}_{i}=\left[\begin{array}{lll}y_{i 1} & y_{i 2} & y_{i 3}\end{array}\right]^{\prime}$ is the vector of responses for the 3 traits on record or animal $i, i=1,2,3, \ldots, n$. Furthermore, $\boldsymbol{\beta}=\left[\begin{array}{lll}\boldsymbol{\beta}_{1}{ }^{\prime} & \boldsymbol{\beta}_{2}{ }^{\prime} & \boldsymbol{\beta}_{3}{ }^{\prime}\end{array}\right] '$ is the vector of fixed effects connected to $\mathbf{y}_{i}$ by known incidence matrix $\mathbf{X}_{i}=\left(\mathbf{I}_{3} \otimes \mathbf{x}_{i}^{\prime}\right)$ such that $\boldsymbol{\beta}_{j}$ denotes the subvector of fixed effects for trait $j(j=1,2,3)$. Note that $\otimes$ denotes the Kronecker product such that we assume the same fixed effects incidence row vector $\mathbf{x}_{i}^{\prime}$ for each of the 3 traits for ease of presentation, although further generalization is possible. Similarly, $\mathbf{u}=\left[\begin{array}{lll}\mathbf{u}_{.1}^{\prime} & \mathbf{u}_{.2}^{\prime} & \mathbf{u}_{.3}^{\prime}\end{array}\right]^{\prime}$ is the vector for animal genetic effects connected to $\mathbf{y}_{i}$ by known incidence matrix $\mathbf{Z}_{i}=\left(\mathbf{I}_{3} \otimes \mathbf{z}_{i}^{\prime}\right)$ such that $\mathbf{u}_{. j}=\left\{u_{i j}\right\}_{i=1}^{n}$ denotes the subvector of random genetic effects on trait $j$ for all $n$ animals. Hence, we also assume the same random effects incidence row vector $\mathbf{z}_{i}^{\prime}$ for each of the 3 traits, although again further generalization is possible. To further simplify presentation, we specifically focus on the situation where there is 1 record per animal, and genetic merit is explicitly modeled only for animals having records, although extensions to the more common situation where genetic evaluations are also desired on animals without any records or multiple records is readily apparent. Finally, $\mathbf{e}_{i}=\left[\begin{array}{lll}e_{i 1} & e_{i 2} & e_{i 3}\end{array}\right]^{\prime}$ denotes the sub-vector of residuals for the 3 traits on animal $i$. Now $\mathbf{e}_{i}$ is assumed to be independently multivariate normal across animals; that is, $\mathbf{e}_{i} \sim M V N\left(\mathbf{0}, \mathbf{R}_{i}\right)$, where $\mathbf{R}_{i}$ denotes the $3 \times 3$ residual (co)variance matrix among 3 traits specific for animal $i$.

Now $\mathbf{u}$ can be alternatively reordered by traits within animals; i.e., $\mathbf{u}=\left[\begin{array}{llll}\mathbf{u}_{1} . & \mathbf{u}_{2} & \cdots & \mathbf{u}_{n}\end{array}\right]$ with $\mathbf{u}_{i .}=\left\{u_{i j}\right\}_{j=1}^{3}$ denoting the vector of random genetic effects for the 3 traits on animal $i$. Because of potential correlations between animal effects due to, for example, the numerator relationship matrix $\mathbf{A}$, the breeding values $\mathbf{u}_{i}$ and $\mathbf{u}_{i^{\prime}}$ for animals $i$ and $i^{\prime}\left(i \neq i^{\prime}\right)$ are not necessarily independent of each other. To flexibly allow for such correlation as well as for subject-specific genetic trait variances and covariances as developed later, we invoke a Cholesky decomposition on $\mathbf{A}$, writing $\mathbf{A}=\mathbf{C C}$ '. For clarity of presentation, we specify the dimension of $\mathbf{A}$ as $n \times n$ such that it only pertains to relationships between animals with records; nevertheless, its construction should be suitably based on all available ancestor information. In other words, we define $\mathbf{A}$ as a subset of a larger numerator relationship matrix that involves both animals with records and their ancestors. Nevertheless, if genetic evaluations are also desired on ancestors, the dimensions of both $\mathbf{A}$ and $\mathbf{z}_{i}^{\prime} \forall i$ can be augmented accordingly. We define $\mathbf{u}_{. j}^{*}$ using $\mathbf{u}_{. j}=\mathbf{C u}_{. j}{ }^{*}$ 
such that $\mathbf{u}^{*}=\left[\begin{array}{llll}\mathbf{u}_{.1}^{*} & \mathbf{u}_{.2}^{*} & \mathbf{u}_{.3}^{*}\end{array}\right]^{\prime}$ is ordered by animals within traits in the same manner as $\mathbf{u}=\left[\begin{array}{lll}\mathbf{u}_{.1}^{\prime} & \mathbf{u}_{.2}^{\prime} & \mathbf{u}_{.3}^{\prime}\end{array}\right]^{\prime}$. Alternatively, if elements of $\mathbf{u}^{*}$ are ordered by elements traits within animals; that is, $\mathbf{u}^{*}=\left[\begin{array}{llll}\mathbf{u}_{1 .}^{*} & \mathbf{u}_{2 .}^{*} & \cdots & \mathbf{u}_{n .}^{*}\end{array}\right]$, then we specify $\mathbf{u}_{i .}^{*}=\left\{u_{i j}^{*}\right\}_{j=1}^{3} \stackrel{\text { independent }}{\sim} \operatorname{MVN}\left(\mathbf{0}, \mathbf{G}_{i}\right)$ for the 3 traits on animal $i$. That is, $\mathbf{G}_{i}$ denotes the $3 \times 3$ genetic (co)variance matrix among the 3 traits being unique to the environmental or management circumstances peculiar to animal $i$. It is important to further clarify, using results from Kennedy et al. (1988), that $\mathbf{u}_{i \text {. }}^{*}$ actually defines the genetic merit on the 3 traits for animal $i$ only if it is a base population animal, whereas $\mathbf{u}_{i \text {. }}^{*}$ defines the Mendelian sampling effects on the 3 traits for animal $i$ if it is a not a base population animal. We thereby rewrite Equation [1] as

$$
\mathbf{y}_{i}=\mathbf{X}_{i} \boldsymbol{\beta}+\mathbf{Z}_{i}\left(\mathbf{I}_{3} \otimes \mathbf{C}\right) \mathbf{u}^{*}+\mathbf{e}_{i}=\mathbf{X}_{i} \boldsymbol{\beta}+\mathbf{Z}_{i}^{*} \mathbf{u}^{*}+\mathbf{e}_{i},[2]
$$

with $\mathbf{Z}_{i}^{*}=\mathbf{Z}_{i}\left(\mathbf{I}_{3} \otimes \mathbf{C}\right)=\mathbf{I}_{3} \otimes \mathbf{z}_{i}^{*_{i}^{\prime}}$, such that $\mathbf{z}_{i}^{*^{\prime}}=\mathbf{z}_{i}^{\prime} \mathbf{C}$, and all other terms defined as before.

Mirroring what was presented previously, albeit in a nongenetic context (Bello et al., 2010) and further extending $\mathrm{Lu}$ et al. (2015), we write the Cholesky decomposition for $\mathbf{G}_{i}$ as $\mathbf{G}_{i}=\left(\mathbf{I}_{3}-\mathbf{T}_{i}^{u}\right)^{-1} \boldsymbol{\Delta}_{i}^{u}\left(\mathbf{I}_{3}-\mathbf{T}_{i}^{u}\right)^{-1}$ ', where $\mathbf{I}_{3}$ is an identity matrix of order 3, $\Delta_{i}^{u}=\operatorname{diag}\left(\sigma_{u_{(1) i}}^{2}, \sigma_{u_{(2 \mid 1) i}}^{2}, \sigma_{u_{(3 \mid 1,2) i}}^{2}\right)$ is a diagonal matrix of sequentially conditional variance components (VC) and

$$
\mathbf{T}_{i}^{u}=\left[\begin{array}{ccc}
0 & 0 & 0 \\
\phi_{(21) i}^{u} & 0 & 0 \\
\phi_{(31) i}^{u} & \phi_{(32) i}^{u} & 0
\end{array}\right]
$$

is a lower triangular matrix such that $\Delta_{i}^{u}$ and $\mathbf{T}_{i}^{u}$ are modeled as being potentially unique to the environmental or management circumstances pertaining to animal $i$. Now $\Delta_{i}^{u}=\operatorname{var}\left(\boldsymbol{\delta}_{i}\right)$ where $\boldsymbol{\delta}_{i}=\left[\begin{array}{lll}u_{i(1)}^{*} & u_{i(2 \mid 1)}^{*} & u_{i(3 \mid 1,2)}^{*}\end{array}\right]$ is based on the specification $\mathbf{u}_{i .}^{*}=\mathbf{T}_{i}^{u} \mathbf{u}_{i .}^{*}+\boldsymbol{\delta}_{i}$ whose elements can be written recursively (Lu et al., 2015) as

$$
\begin{gathered}
u_{i 1}^{*}=u_{i(1)}^{*}, \\
u_{i 2}^{*}=\phi_{(21) i}^{u} u_{i(1)}^{*}+u_{i(2 \mid 1)}^{*}, \\
u_{i 3}^{*}=\phi_{(31) i}^{u} u_{i 1}^{*}+\phi_{(32) i}^{u} u_{i 2}^{*}+u_{i(3 \mid 1,2)}^{*} .
\end{gathered}
$$

For instance, $u_{i(2 \mid 1)}^{*}$ is used to represent the genetic effect of MBW conditional on MILKE for animal $i$, whereas $u_{i(3 \mid, 2)}^{*}$ pertains to the genetic effect of DMI conditional on MILKE and MBW and which we have previously proposed as the genetic effect for FE for animal $i$ ( $\mathrm{Lu}$ et al., 2015). Similarly, we define $\sigma_{u(3 \mid, 2) i}^{2}$ as the Mendelian sampling genetic variance of $\mathrm{FE}$ for animal $i$. Lu et al. (2015) referred to $\phi_{(21) i}^{u}, \phi_{(31) i}^{u}$, and $\phi_{(32) i}^{u}$ as PE at the genetic level. We further extend their work using Bello et al. (2010) to allow for the possibility that these genetic PE are potentially heterogeneous across cows as manifested by the additional subscript $i$.

$\mathrm{Lu}$ et al. (2015) likewise demonstrated $\mathbf{R}_{i}=\left(\mathbf{I}_{3}-\mathbf{T}_{i}^{e}\right)^{-1} \boldsymbol{\Delta}_{i}^{e}\left(\mathbf{I}_{3}-\mathbf{T}_{i}^{e}\right)^{-1}{ }^{\prime}, \quad$ where $\boldsymbol{\Delta}_{i}^{e}=\operatorname{diag}\left(\sigma_{e_{(1) i}}^{2}, \sigma_{e_{(2 \mid 1) i}}^{2}, \sigma_{e_{(31,2) i}}^{2}\right)$ is a diagonal matrix and

$$
\mathbf{T}_{i}^{e}=\left[\begin{array}{ccc}
0 & 0 & 0 \\
\phi_{(21) i}^{e} & 0 & 0 \\
\phi_{(31) i}^{e} & \phi_{(32) i}^{e} & 0
\end{array}\right]
$$

is a lower triangular matrix except that here both $\Delta_{i}^{e}$ and $\mathbf{T}_{i}^{(e)}$ are potentially unique to the environmental circumstances peculiar to animal $i$ following Bello et al. (2010). Now $\Delta_{i}^{e}$ is the covariance matrix of $\varepsilon_{i}=\left[\begin{array}{lll}e_{(1) i} & e_{(2 \mid 1) i} & e_{(3 \mid 1,2) i}\end{array}\right]$ ' as defined within $\mathbf{e}_{i .}=\mathbf{T}_{i}^{e} \mathrm{e}_{i .}+\varepsilon_{i}$ whose elements can be demonstrated to be written recursively, using Lu et al. (2015), as

$$
\begin{gathered}
e_{i 1}=e_{i(1)}, \\
e_{i 2}=\phi_{(21) i}^{e} e_{i 1}+e_{i(2 \mid 1)}, \\
e_{i 3}=\phi_{(31) i}^{e} e_{i 1}+\phi_{(32) i}^{e} e_{i 2}+e_{i(3 \mid 1,2)} .
\end{gathered}
$$

Here, we refer to $\phi_{(21) i}^{e}, \phi_{(31) i}^{e}$, and $\phi_{(32) i}^{e}$ as PE at the residual level, again being unique to animal $i$.

Equations [3c] and [4c] are particularly important for defining FE in that they specify the key relationships between the energy source (DMI) and energy sink traits (MILKE and MBW) at both the genetic (i.e., $\phi_{(31) i}^{u}$, and $\left.\phi_{(32) i}^{u}\right)$ and residual $\left(\phi_{(31) i}^{e}\right.$ and $\left.\phi_{(32) i}^{e}\right)$ levels. For example, $\phi_{(31) i}^{u}$ defines the change in $u_{i 3}$ for every unit change in $u_{i 1}$ holding constant $u_{i 2}$, whereas $\phi_{(32) i}^{u}$ defines the change in $u_{i 3}$ for every unit change in $u_{i 2}$ holding constant $u_{i 1}$ on animal $i$. Furthermore, $\phi_{(31) i}^{e}$ and $\phi_{(32) i}^{e}$ are similarly in- 
terpreted but at the residual or nongenetic level with respect to animal $i$. In essence, heterogeneous $\mathrm{PE}$ at the genetic or residual levels indirectly imply heterogeneous genetic and residual correlations between traits across animals as determined by different environments and management factors.

As in Bello et al. (2010), we specify a structural mixed effects model on each of these PE. For example, at the genetic level, we write

$$
\phi_{(r) i}^{u}=\mathbf{x}_{(r) i}^{u}{ }^{\prime} \gamma_{(r)}^{u}+\mathbf{z}_{(r) i}^{u}{ }^{\prime} \mathbf{m}_{(r)}^{u},
$$

with $r=21,31$, or 32 denoting indices representing $\mathrm{PE}$ relationships between MBW and MILKE $(r=21)$, between DMI and MILKE $(r=31)$ and between DMI and MBW $(r=32)$ as per Equations [3b] and [3c]. Here, $\gamma_{(r)}^{u}$ represents a $p_{u(r)} \times 1$ vector of unknown fixed effects connected to $\phi_{(r) i}^{u}$ by known incidence row vector $\mathbf{x}_{(r) i}^{u}{ }^{\prime}$, whereas $\mathbf{m}_{(r)}^{u} \sim N\left(\mathbf{0}, \mathbf{I} \sigma_{m_{u}(r)}^{2}\right)$ represents a $q_{u(r)} \times 1$ vector of unknown random effects connected to $\phi_{(r) i}^{u}$ by known incidence row vector $\mathbf{z}_{(r) i}^{u}$ '. In this context, fixed effects pertain to those effects characterized by either noninformative or vaguely informative prior distributions that do not presume exchangeability among the elements of $\gamma_{(r)}^{u}$. Strategically, random effects specifications are often best used for factors characterized by a large number of subclasses, with each subclass characterized by relatively limited data (Bello et al., 2010).

We similarly specify a structural mixed effects model for the residual $\mathrm{PE} \phi_{(21) i}^{e}, \phi_{(31) i}^{e}$, and $\phi_{(32) i}^{e}$ specific to observation $i$

$$
\phi_{(r) i}^{e}=\mathbf{x}_{(r) i}^{e}{ }^{\prime} \gamma_{(r)}^{e}+\mathbf{z}_{(r) i}^{e}{ }^{\prime} \mathbf{m}_{(r)}^{e},
$$

with $r=21,31$, or 32 as before as per Equations [4b] and $[4 \mathrm{c}]$. Here, $\boldsymbol{\gamma}_{(r)}^{e}$ denotes a $p_{e(r)} \times 1$ vector of fixed effects connected to $\phi_{(r) i}^{e}$ by known incidence row vector $\mathbf{x}_{(r) i}^{e}$, whereas $\mathbf{m}_{(r)}^{e} \sim N\left(\mathbf{0}, \mathbf{I} \sigma_{m_{e}(r)}^{2}\right)$ represent a $q_{e(r)} \times 1$ vector of random effects connected to $\phi_{(r) i}^{e}$ by $\mathbf{z}_{(r) i}^{e}$ ' where terms are similarly interpreted as with genetic PE.

Following Bello et al. (2010), each VC specific to animal $i$ in $\Delta_{i}^{u}=\operatorname{diag}\left(\sigma_{u_{(1) i}}^{2}, \sigma_{u_{(2|1|)}}^{2}, \sigma_{u_{(3 \mid 1,2) i}}^{2}\right)$ is also specified as a function of environmental or management factors in a structural mixed effects model:

$$
\log \left(\sigma_{u(s) i}^{2}\right)=\mathbf{x}_{(s) i}^{u}{ }^{\prime} \log \left(\boldsymbol{\tau}_{(s)}^{u}\right)+\mathbf{z}_{(s) i}^{u}{ }^{\prime} \log \left(\mathbf{v}_{(s)}^{u}\right)
$$

where $s=1,2 \mid 1$, and $3 \mid 1,2$ is used to denote, respectively, the genetic variance for MBW, the genetic variance for MBW conditional on MILKE, and the genetic variance for DMI conditional on MILKE and MBW. Here $\boldsymbol{\tau}_{(s)}^{u}$ is a $p_{u(s)} \times 1$ vector of fixed effects connected to $\sigma_{u(s) i}^{2}$ by known incidence row vector $\mathbf{x}_{(s) i}^{u}$, whereas $\mathbf{v}_{(s)}^{u}$ is a $q_{u(s)} \times 1$ vector of random effects connected to $\sigma_{u(s) i}^{2}$ by known incidence row vector $\mathrm{z}_{(s) i}^{u}$. Independent inverted Gamma priors $I G\left(\eta_{(s)}^{u}, \eta_{(s)}^{u}-1\right)$ are assigned to each element of $\mathbf{v}_{(s)}^{u}=\left\{v_{(s) d}^{u}\right\}_{d=1}^{q_{u(s)}}$ such that $\mathrm{E}\left(v_{(s) d}^{u}\right)=1$ with coefficient of variation of

$$
\mathrm{CV}\left(\mathbf{v}_{(s)}^{u}\right)=\frac{1}{\sqrt{\eta_{(s)}^{u}-2}}
$$

as previously demonstrated by Kizilkaya and Tempelman (2005) and Bello et al. (2010). That is, $\operatorname{CV}\left(\mathbf{v}_{(s)}^{u}\right)$ characterizes the standard deviation of subclass-specific variances expressed relative to the average subclass variance.

Finally, the $\mathrm{VC}$ in $\Delta_{i}^{e}=\operatorname{diag}\left(\sigma_{e_{(1) i}}^{2}, \sigma_{e_{(2 \mid 1) i}}^{2}, \sigma_{e_{(3 \mid 1,2) i}}^{2}\right)$ are similarly modeled with a mixed effects specification:

$$
\log \left(\boldsymbol{\sigma}_{e(s) i}^{2}\right)=\mathbf{x}_{(s) i}^{e}{ }^{\prime} \log \left(\boldsymbol{\tau}_{(s)}^{e}\right)+\mathbf{z}_{(s) i}^{e}{ }^{\prime} \log \left(\mathbf{v}_{(s)}^{e}\right)
$$

where $s=1,2 \mid 1$, and $3 \mid 1,2$ is used to denote, respectively, the residual variance for MBW, the residual variance for MBW conditional on MILKE, and the residual variance for DMI conditional on MILKE and MBW. Here $\boldsymbol{\tau}_{(s)}^{e}$ denotes a $p_{e(s)} \times 1$ vector of fixed effects connected to $\sigma_{e(s) i}^{2}$ by known incidence row vector $\mathbf{x}_{(s) i}^{e}$, whereas $\mathbf{v}_{(s)}^{e}$ represents a $q_{e(s)} \times 1$ vector of random effects connected to $\sigma_{e(s) i}^{2}$ by known incidence row vector $\mathbf{z}_{(s) i}^{e}$. Here, elements of $\mathbf{v}_{(s)}^{e^{\prime}}=\left\{v_{(s) l}^{e}\right\}_{l=1}^{q_{e(s)}}$ are assumed to be random independent draws from $I G\left(\eta_{(s)}^{e}, \eta_{(s)}^{e}-1\right)$ such that $\mathrm{E}\left(v_{(s) l}^{e}\right)=1$ and $\operatorname{CV}\left(\mathbf{v}_{(s)}^{e}\right)=1 /\left(\sqrt{\eta_{(s)}^{e}-2}\right)$.

Following Bello et al. (2010) and Kizilkaya and Tempelman (2005), vaguely informative priors are specified on $\eta_{(s)}^{e}$ and $\eta_{(s)}^{u}$ as follows:

$$
\eta_{(s)}^{u} \sim \mathrm{p}\left(\eta_{(s)}^{u}\right)=\frac{1}{\left(1+\eta_{(s)}^{u}\right)^{2}},
$$




$$
\eta_{(s)}^{e} \sim \mathrm{p}\left(\eta_{(s)}^{e}\right)=\frac{1}{\left(1+\eta_{(s)}^{e}\right)^{2}},
$$

for $s=1,2 \mid 1$, and $3 \mid 1,2$.

For all analyses in this paper, flat unbounded priors are specified on $\boldsymbol{\beta}_{1}, \boldsymbol{\beta}_{2}, \boldsymbol{\beta}_{3}, \boldsymbol{\gamma}_{(21)}^{u}, \boldsymbol{\gamma}_{(31)}^{u}, \boldsymbol{\gamma}_{(32)}^{u}, \boldsymbol{\gamma}_{(21)}^{e}, \boldsymbol{\gamma}_{(31)}^{e}$, $\boldsymbol{\tau}_{(1)}^{u}, \boldsymbol{\tau}_{(2 \mid 1)}^{u}, \boldsymbol{\tau}_{(3 \mid 1,2)}^{u}, \boldsymbol{\tau}_{(1)}^{e}, \boldsymbol{\tau}_{(2 \mid 1)}^{e}, \boldsymbol{\tau}_{(3 \mid 1,2)}^{e}, \boldsymbol{\sigma}_{m_{u}(21)}^{2}, \boldsymbol{\sigma}_{m_{u}(31)}^{2}, \boldsymbol{\sigma}_{m_{u}(32)}^{2}$, $\boldsymbol{\sigma}_{m_{e}(21)}^{2}, \boldsymbol{\sigma}_{m_{e}(31)}^{2}$, and $\boldsymbol{\sigma}_{m_{e}(32)}^{2}$, as also adapted by Bello et al. (2010), although vaguely or even rather informative priors could be specified as well. Due to these flat prior specifications, it is imperative to invoke identifiability restrictions on $\gamma_{(21)}^{u}, \gamma_{(31)}^{u}, \gamma_{(32)}^{u}, \gamma_{(21)}^{e}, \gamma_{(31)}^{e}, \gamma_{(32)}^{e}, \boldsymbol{\tau}_{(1)}^{u}, \boldsymbol{\tau}_{(2 \mid 1)}^{u}$, $\boldsymbol{\tau}_{(3 \mid 1,2)}^{u}, \boldsymbol{\tau}_{(1)}^{e}, \boldsymbol{\tau}_{(2 \mid 1)}^{e}$, and $\boldsymbol{\tau}_{(3 \mid 1,2)}^{e}$ similar to those commonly invoked for fixed classification factors (i.e., $\boldsymbol{\beta}_{1}, \boldsymbol{\beta}_{2}$, and $\boldsymbol{\beta}_{3}$ ) in classical linear model specifications. In that regard, we use the corner parameterization (Clayton, 1996; Kizilkaya and Tempelman, 2005) also referred to as the set-to-zero restriction (Milliken and Johnson, 2009) and as also used by Bello et al. (2010) whereby an overall intercept is specified and the effect corresponding to one arbitrarily chosen level of each fixed effects factor is ignored or "zeroed out."

Full conditional densities of all unknown (hyper) parameters as required for conducting Markov Chain Monte Carlo (MCMC) Bayesian analysis are provided in Supplemental File S1 (https://doi.org/10.3168/ jds.2016-11491).

\section{Application to Dairy Consortium Data}

Data Source and Editing. A data set of MILKE, DMI, and MBW on 5,088 Holstein cows was collected on 13 research stations and studies from the United Kingdom, the Netherlands, Canada, and the United States; data sources and editing procedures used for this study are extensively characterized elsewhere (Berry et al., 2014; Lu et al., 2015; Tempelman et al., 2015; Manzanilla-Pech et al., 2016). Phenotypes on all 3 traits were further condensed into 42 -d records by taking the average of the first 6 weekly records on all 3 traits between 50 and 200 DIM. If data from multiple lactations were available on individual cows, only the earlier lactation was chosen. One research station was in Canada, being the University of Alberta (AB). Six stations were in the United States, specifically, Iowa State University (ISU), Michigan State University (MSU), the University of Florida (UF), the University of Wisconsin-Madison (UW), the USDA Dairy Forages Research Center (USDFRC) in Madison, Wisconsin, and the USDA Beltsville Agricultural Re- search Center (BARC) in Beltsville, Maryland. Four research stations were in the Netherlands including an experimental herd 't Gen (TGEN) in Lelystad, the Nij Bosma Zathe (NBZ) herd located near Leeuwarden, a third study (ZOM) based on the work by Zom et al. (2012); and a compilation of studies (NLN) based on data collected from various nutritional experiments, all of which were previously characterized in detail by Tempelman et al. (2015). The remaining 2 herds were from the United Kingdom, the Langhill (LAN) farm near Edinburgh from 1992 to 2001 and from the Scottish Agricultural College (SAC) Dairy Research Centre based at Crichton Royal Farm near Dumfries with data collection from 2003 to 2011; again, these data are also described extensively elsewhere (Tempelman et al., 2015). A summary of the number of cows by research station/study is provided in Table 1 .

The specification for the full hierarchical Bayesian model included the fixed effects of research station and parity (primiparous versus multiparous) for each of $\boldsymbol{\tau}_{(1)}^{u}$, $\boldsymbol{\tau}_{(2 \mid 1)}^{u}, \boldsymbol{\tau}_{(3 \mid 1,2)}^{u}, \boldsymbol{\tau}_{(1)}^{e}, \boldsymbol{\tau}_{(2 \mid 1)}^{e}$, and $\boldsymbol{\tau}_{(3 \mid 1,2)}^{e}$. Research station, parity, and the linear and quadratic effects of DIM were specified in $\gamma_{(21)}^{u}, \gamma_{(31)}^{u}, \gamma_{(32)}^{u}, \gamma_{(21)}^{e}, \gamma_{(31)}^{e}$, and $\gamma_{(32)}^{e}$. Finally, research station, parity, ration with station, and up to 4th order polynomial effects of DIM were specified in $\boldsymbol{\beta}_{1}, \boldsymbol{\beta}_{2}$, and $\boldsymbol{\beta}_{3}$. Ration within station was specified in all cases as the single random effects factor for each of $\mathbf{m}_{(r)}^{u}$, $\mathbf{m}_{(r)}^{e}, \mathbf{v}_{(s)}^{u}$, and $\mathbf{v}_{(s)}^{e}$. Given that there were a total of 271

Table 1. Distribution of cows by region ${ }^{1}$

\begin{tabular}{llc}
\hline Region & Station & $\begin{array}{c}\text { No. of cows } \\
\text { with phenotypes }\end{array}$ \\
\hline Canada & AB & 236 \\
United States & UF & 175 \\
& ISU & 742 \\
MSU & 158 \\
UW & 237 \\
USDFRC & 565 \\
BARC & 286 \\
The Netherlands & NGEN & 569 \\
& ZOMZ & 99 \\
& NLN & 661 \\
United Kingdom & LAN & 329 \\
& SAC & 590 \\
Overall & & 441 \\
1AB = University of Alberta; UF = University of Florida; ISU = Iowa \\
State University; MSU = Michigan State University; UW = University \\
of Wisconsin-Madison; USDFRC = USDA Dairy Forages Research \\
Center; BARC = USDA Beltsville Agricultural Research Center; \\
TGEN = 't Gen experimental herd, Lelystad, the Netherlands; NBZ \\
$=$ Nij Bosma Zathe, Leeuwarden, the Netherlands; ZOM = data based \\
on the work of Zom et al. (2012); NLN = compilation of studies previ- \\
ously characterized by Tempelman et al. (2015); LAN = Langhill farm, \\
Edinburgh, UK; SAC = Scottish Agricultural College.
\end{tabular}


rations across the 13 research stations, it was more important to specify these ration effects as random at the deepest levels of the model hierarchy (i.e., for PE and $\mathrm{VC}$ ) to facilitate efficient borrowing of information across rations. Conversely, treating ration effects as fixed in $\boldsymbol{\beta}_{1}, \boldsymbol{\beta}_{2}$, and $\boldsymbol{\beta}_{3}$ was deemed to be feasible because these effects are specified closer to the data $(\mathbf{y})$ in the model hierarchy. To account for changes in energy balance, the average daily change in $\mathrm{BW}(\boldsymbol{\Delta B W})$ was fitted as a covariate in $\boldsymbol{\beta}_{1}, \boldsymbol{\beta}_{2}$, and $\boldsymbol{\beta}_{3}$ as well.

Model Comparison. Model comparison and selection in hierarchical Bayesian inference is often formally based on the deviance information criterion (DIC; Spiegelhalter et al., 2002) as well as on the relative sizes of the posterior $z$-scores; that is, the ratio of the posterior means to their posterior standard deviations (Gelman et al., 2012). The absolute values of these posterior $z$-scores can be used in Wald-like tests to ascertain, for example, whether or not they exceed 1.96 in absolute value for a type I error rate of $5 \%$, assuming the marginal posterior density of the corresponding parameter is reasonably symmetric. The DIC has previously been used in a stepwise manner to infer evidence for fixed and random sources of heterogeneity on $\mathrm{VC}$ and PE within a 2-trait model by Bello et al. (2012). However, adapting their stepwise process would be far more onerous for our 3-trait model given the need for an assessment of which fixed or random effects factors to keep for each of 12 different mixed model subsets (i.e., 3 separate models for each of Equations [5], [6], [7], and [8]) beyond the classical mixed model specification in Equation [1]. Thus, we only compared 5 models starting at one extreme from a purely homoscedastic MT model with homogeneous PE and VC at all levels $\left(\mathrm{M}_{0}\right)$ as developed and used previously in Lu et al. (2015) to the proposed fully heteroscedastic MT model with heterogeneous PE and VC, based on all structural mixed effects model specifications as detailed in this paper $\left(\mathrm{M}_{4}\right)$. The remaining models evaluated were $\left(\mathrm{M}_{1}\right)$ fixed effects (i.e., without random effects) modeling on both genetic and residual $\mathrm{PE}$ with homogeneous genetic and residual $\mathrm{VC},\left(\mathrm{M}_{2}\right)$ fixed effects modeling on genetic and residual $\mathrm{PE}$ and on genetic and residual $\mathrm{VC}$, and $\left(\mathrm{M}_{3}\right)$ full mixed effects modeling on $\mathrm{VC}$ with only fixed effects modeling on PE.

Cross-Validation Assessment. To assess the relative importance of modeling heterogeneous genetic and residual $\mathrm{VC}$ and $\mathrm{PE}$, we assessed the cross-validation performance of our proposed model $\left(\mathrm{M}_{4}\right)$ relative to the more conventional MT mixed model $\left(\mathrm{M}_{0}\right)$ of $\mathrm{Lu}$ et al. (2015). More specifically, a 5-fold cross-validation study was conducted by merely randomly partitioning the entire data set into 5 equally sized subsets such that for each of the folds, 4 subsets were chosen as training data with the remaining subset chosen as validation data. The performance of the 2 models were compared with respect to cross-validation predictive accuracy defined as the correlation between true DMI and prediction in the validation data sets based on estimates derived from the analyses of the training data sets.

Marginal Means. All reported estimates were based on the full model $\left(\mathrm{M}_{4}\right)$, determined to be the best fitting of those evaluated. To facilitate interpretation, however, estimates of all fixed effects were re-expressed to a marginal mean or least squares means basis as also popularized by SAS software (Littell et al., 2002; Milliken and Johnson., 2009); further illustration in the context of our MT model is presented by Bello et al. (2010) and Bello et al. (2012). For example, linear combinations of $\gamma_{(21)}^{u}, \gamma_{(31)}^{u}, \gamma_{(32)}^{u}, \gamma_{(21)}^{e}, \gamma_{(31)}^{e}$, and $\gamma_{(32)}^{e}$ were used to estimate marginal genetic and residual PE relationships for the various research stations, averaged across levels of the remaining factor(s) (i.e., parity) and at the midpoint covariate values (i.e., DIM $=125 \mathrm{~d}$ ). Marginal mean linear combinations of $\boldsymbol{\tau}_{(1)}^{u}, \boldsymbol{\tau}_{(2 \mid 1)}^{u}, \boldsymbol{\tau}_{(3 \mid 1,2)}^{u}$, $\boldsymbol{\tau}_{(1)}^{e}, \boldsymbol{\tau}_{(2 \mid 1)}^{e}$, and $\boldsymbol{\tau}_{(3 \mid 1,2)}^{e}$ were exponentiated to the $\mathrm{VC}$ scale in a manner similar to that described by Bello et al. (2010). Pairwise comparisons between marginal posterior means were conducted between levels of each fixed effects factor (i.e., stations and parity) using 2-tailed Bayesian $P$-values in the manner described previously by Bello et al. (2010).

\section{Simulation Study}

We realize that our proposed Bayesian MT model on 3 traits is massively hierarchical in nature because it entails a total of 15 different mixed model specifications, including 3 within the basic first stage specification for $\mathbf{y}_{i}$ in Equation [1]; one for each of $\phi_{(21) i}^{u}, \phi_{(31) i}^{u}$, and $\phi_{(32) i}^{u}$ in Equation [5]; one for each of $\phi_{(21) i}^{e}, \phi_{(31) i}^{e}$, and $\phi_{(32) i}^{e}$ in Equation [6]; one for each of $\sigma_{u(1) i}^{2}, \sigma_{u(2 \mid 1) i}^{2}$, and $\sigma_{u(3 \mid 1,2) i}^{2}$ in Equation [7]; and one for each of $\sigma_{e(1) i}^{2}, \sigma_{e(2 \mid 1) i}^{2}$, and $\sigma_{e(3 \mid 1,2) i}^{2}$ in Equation [8]. Hence, similar to Bello et al. (2010), we used a simulation study to validate the proposed model and Bayesian inference strategy as developed elsewhere.

Twenty replicated data sets were simulated based on only the subset of the data and pedigree deriving from the US research stations to facilitate computational tractability. Overall intercept and station effects were generated as fixed effects for $\operatorname{PE}\left(\gamma_{(21)}^{u}, \gamma_{(31)}^{u}, \gamma_{(32)}^{u}, \gamma_{(21)}^{e}\right.$, $\gamma_{(31)}^{e}$, and $\left.\gamma_{(32)}^{e}\right)$ and for the $\operatorname{VC}\left(\tau_{(1)}^{u}, \tau_{(2 \mid 1)}^{u}, \tau_{(3 \mid 1,2)}^{u}, \tau_{(1)}^{e}, \tau_{(2 \mid 1)}^{e}\right.$, and $\left.\tau_{(3 \mid 1,2)}^{e}\right)$, whereas ration within station effects were 
generated as random effects with $\mathrm{VC} \sigma_{m_{u}(21)}^{2}, \sigma_{m_{u}(31)}^{2}$, $\sigma_{m_{u}(32)}^{2}$ for the genetic PE and $\sigma_{m_{e}(21)}^{2}, \sigma_{m_{e}(31)}^{2}$, and $\sigma_{m_{e}(32)}^{2}$ for the residual PE and with hyperparameters $\eta_{(1)}^{u}, \eta_{(2 \mid 1)}^{u}$, and $\eta_{(3 \mid 1,2)}^{u}$ for the genetic $\mathrm{VC}$ and $\eta_{(1)}^{e}, \eta_{(2 \mid 1)}^{e}$, and $\eta_{(3 \mid 1,2)}^{e}$ for the residual VC. True values for these (hyper)parameters were roughly determined as estimates from the analysis of subset of data deriving from the United States and are provided in Table 2. Coverage probabilities of the $95 \%$ highest posterior density (HPD) interval for each parameter or hyper-parameter were based on how many times the HPD included the true values over the 20 replicated data sets.

\section{MCMC Implementation}

For all analyses involving either the dairy or simulated data, trace plots, autocorrelation, and effective sample size (ESS) for samples of all unknown parameters drawn from the posterior density using MCMC were monitored (Brooks and Gelman, 1998) using the $\mathrm{R}$ package Coda (Plummer et al., 2006). To facilitate quicker convergence, starting values were based on regular BLUP/REML analyses. The number of burn-in cycles were 30,000 for the simulation study and 20,000 for the dairy data analysis with convergence being diagnosed by trace plots. After burn-in, every tenth cycle was saved from 100,000 MCMC cycles such that posterior inference was based on 10,000 cycles. This sampling strategy was sufficient to ensure that ESS $>100$ for all hyperparameters.

\section{RESULTS}

\section{Analysis of Dairy Consortium Data}

As indicated previously, only a small subset of all possible models was considered, the simplest model $\left(\mathrm{M}_{0}\right)$ being equivalent to a classical homogeneous (co) variance matrix multiple-trait modeling specification as in Lu et al. (2015). The DIC values were expressed relative to $\mathrm{M}_{\mathrm{o}}$ (i.e., $\mathrm{DIC}=0$ for $\mathrm{M}_{\mathrm{o}}$ ), which was determined to be the worst fitting model. The DIC values became progressively smaller (i.e., better fitting) for increasingly more complex models starting with $\mathrm{M}_{1}$ : fixed effects but no random effects modeling on both genetic and residual PE with no structural modeling at all for $\mathrm{VC}(\mathrm{DIC}=-1,673) ; \mathrm{M}_{2}$ : only fixed effects modeling on both genetic and residual $\mathrm{PE}$ and $\mathrm{VC}(\mathrm{DIC}=-4,305)$; $\mathrm{M}_{3}$ : full mixed effects modeling on $\mathrm{VC}$ with fixed effects modeling only on $\mathrm{PE}(\mathrm{DIC}=-4,633)$; and finally $\mathrm{M}_{4}$ : full mixed effects modeling on both $\mathrm{VC}$ and $\mathrm{PE}$ (DIC = $-5,400)$, the best fitting of all models and upon which all subsequent inferences on the dairy data analysis are based. Clearly then, it was important to structurally fit genetic and residual sources of heterogeneous $\mathrm{PE}$ and VE per Equations [5], [6], [7], and [8], recognizing that DIC differences exceeding 7 are generally considered to represent significant difference in model fit (Spiegelhalter et al., 2002).

\section{Inferences on Factors Influencing PE}

The posterior means (PMEAN) and posterior standard deviations (PSD), 95\% HPD intervals, and ESS for all PE-related inferences on marginal means of station, parity, linear, and quadratic effects of DIM at both genetic $\left(\boldsymbol{\gamma}_{(21)}^{u}, \boldsymbol{\gamma}_{(31)}^{u}\right.$, and $\left.\boldsymbol{\gamma}_{(32)}^{u}\right)$ and residual levels $\left(\gamma_{(21)}^{e}, \gamma_{(31)}^{e}\right.$, and $\left.\gamma_{(32)}^{e}\right)$ as well as $\mathrm{VC}$ for random effects at the genetic $\left(\boldsymbol{\sigma}_{m_{u}(21)}^{2}, \boldsymbol{\sigma}_{m_{u}(31)}^{2}\right.$, and $\left.\boldsymbol{\sigma}_{m_{u}(32)}^{2}\right)$ and residual $\left(\boldsymbol{\sigma}_{m_{e}(21)}^{2}, \boldsymbol{\sigma}_{m_{e}(31)}^{2}\right.$, and $\left.\boldsymbol{\sigma}_{m_{e}(32)}^{2}\right)$ levels are summarized in Supplemental Table S1 (https://doi.org/10.3168/ jds.2016-11491) for MBW|MILKE, in Table 2 for DMI|MILKE, and in Table 3 for DMI|MBW, respectively.

Although we do not particularly highlight results regarding MBW|MILKE in this paper, it is intriguing to note from Supplemental Table S1 (https://doi. org/10.3168/jds.2016-11491) that the genetic PE between the 2 traits were not generally different from 0 for any one research station or for either parity because 0 fell within the $95 \%$ HPD in all cases. Conversely, residual relationships were universally positive. Taken together and using Equation [7] in Lu et al. (2015), these results imply a universally negligible genetic correlation but a positive residual correlation between MBW and MILKE. At any rate, there was also no formal evidence of any heterogeneity in these relationships between research stations or parities based on pairwise comparisons between the corresponding marginal means. Also, no evidence was found of heterogeneous genetic variances but strong evidence was found of heterogeneous residual variances across stations for MILKE (Supplementary Table S2), whereas no evidence was found of any heterogeneous genetic or residual variances for MBW|MILKE across stations (Supplemental Table S3; https://doi.org/10.3168/jds.2016-11491).

Because our focus was on $\mathrm{FE}$, we were particularly interested in PE relationships involving DMI. As a basis for reference, the overall mean PE at the genetic and residual levels were respectively determined to be

$$
\bar{\phi}_{(r)}^{u}=\frac{1}{n} \sum_{i=1}^{n}\left(\mathbf{x}_{(r) i}^{u}{ }^{\prime} \hat{\boldsymbol{\gamma}}_{(r)}^{u}+\mathbf{z}_{(r) i}^{u}{ }^{\prime} \hat{\mathbf{m}}_{(r)}^{u}\right)
$$


HETEROGENEOUS RELATIONSHIPS IN FEED EFFICIENCY

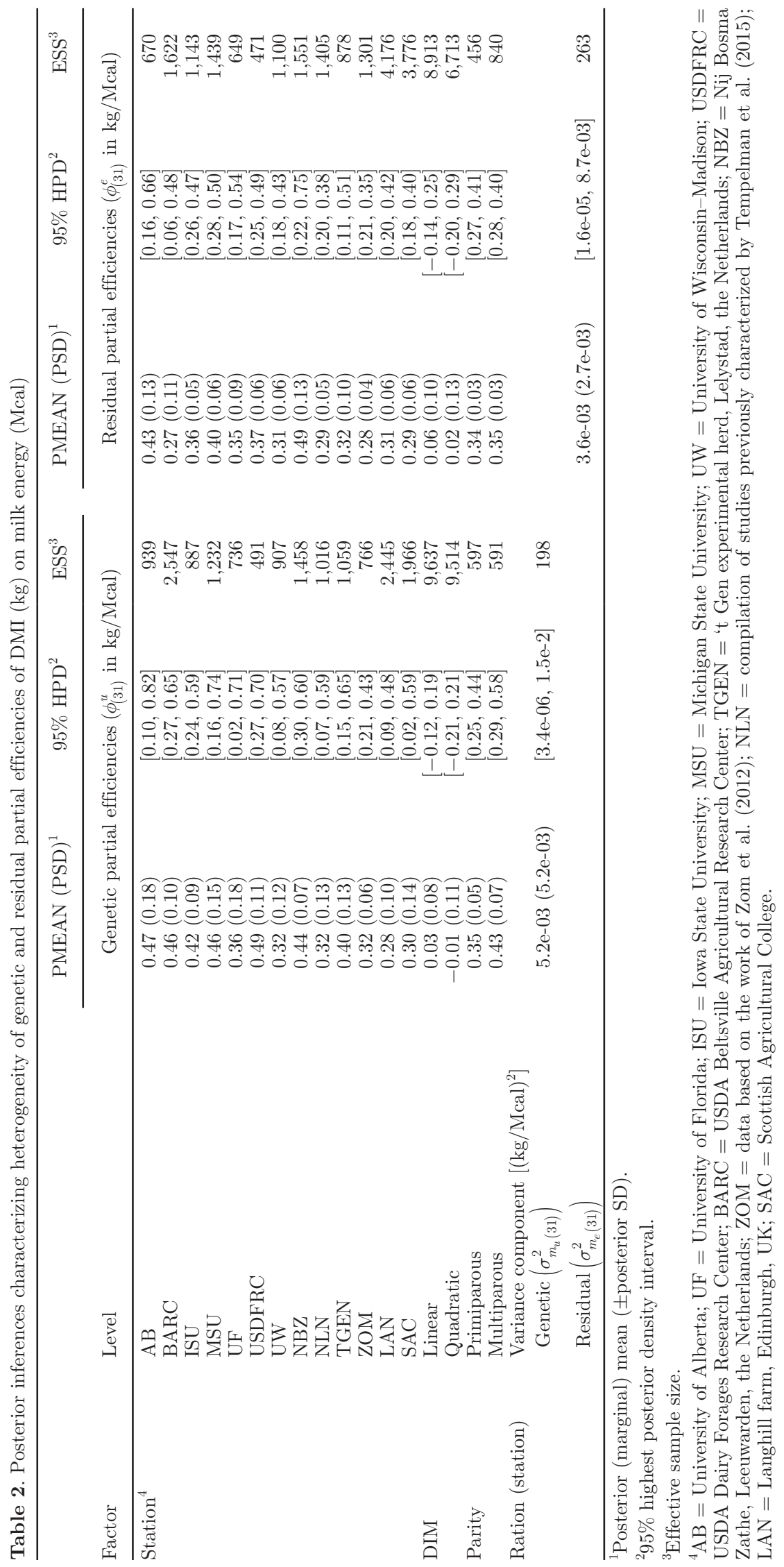


LU ET AL.

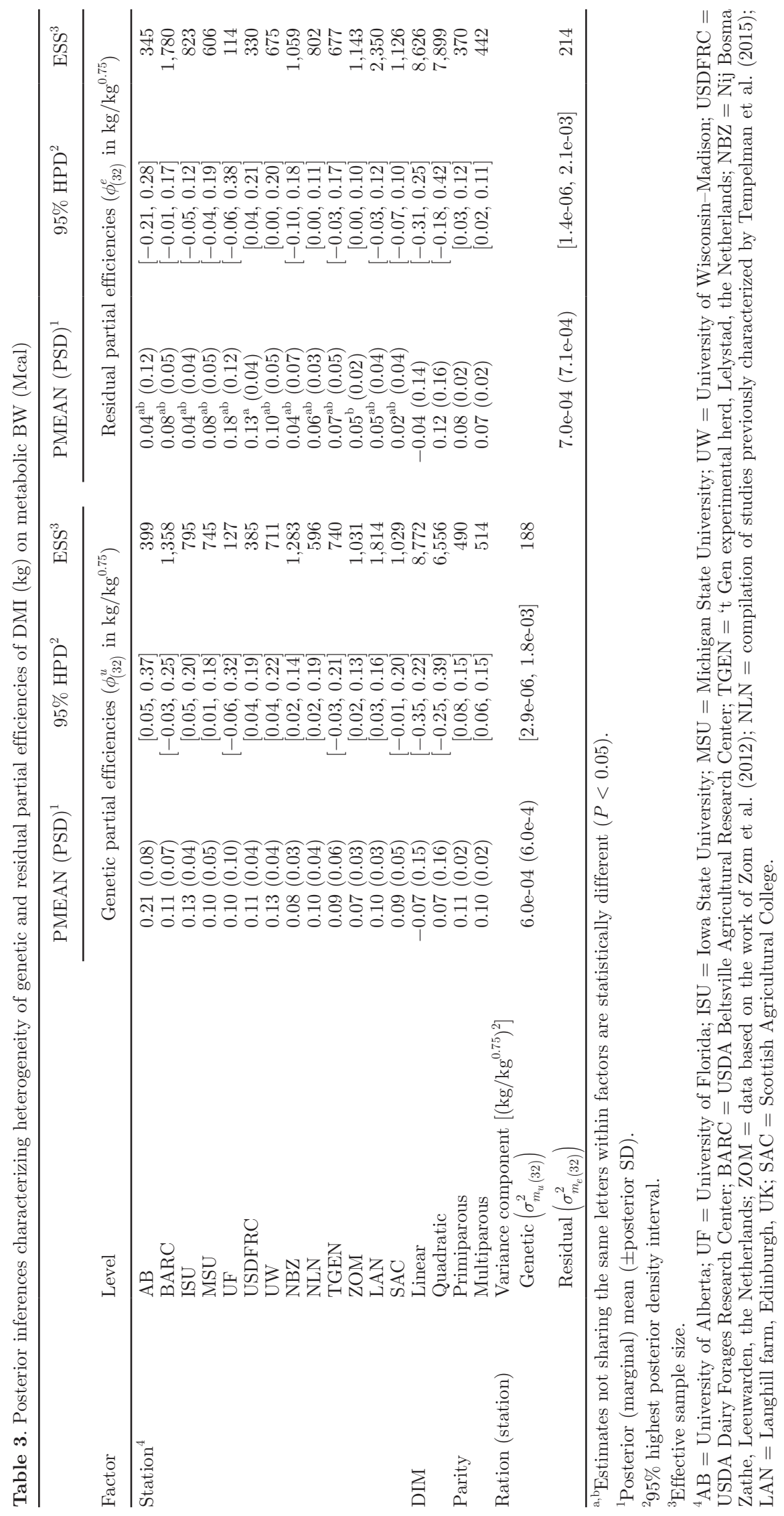


and

$$
\bar{\phi}_{(r)}^{e}=\frac{1}{n} \sum_{i=1}^{n}\left(\mathbf{x}_{(r) i}^{e}{ }^{\prime} \hat{\gamma}_{(r)}^{e}+\mathbf{z}_{(r) i}^{e}{ }^{\prime} \hat{\mathbf{m}}_{(r)}^{e}\right)
$$

where $\hat{\boldsymbol{\gamma}}_{(r)}^{u}, \hat{\mathbf{m}}_{(r)}^{u}, \hat{\boldsymbol{\gamma}}_{(r)}^{e}$, and $\hat{\mathbf{m}}_{(r)}^{e}$ denote the PMEAN of the corresponding parameters for $r=31$ or 32 (i.e., PE of DMI on MILKE and of DIM on MBW, respectively). Specifically, the PMEAN $( \pm \mathrm{PSD})$ of $\bar{\phi}_{(31)}^{u}$ was $0.38( \pm 0.10)$ $\mathrm{kg} /$ Mcal, whereas the PMEAN ( \pm PSD) of $\bar{\phi}_{(32)}^{u}$ was $0.10( \pm 0.05) \mathrm{kg} / \mathrm{kg}^{0.75}$, implying then, for example, the genetic merit of DMI is estimated to increase by $0.38 \mathrm{~kg}$ on average for every megacalorie increase in the genetic merit of MILKE holding constant MBW. At the residual level, the PMEAN $( \pm \mathrm{PSD})$ of $\bar{\phi}_{(31)}^{e}$ was $0.33( \pm 0.06)$ $\mathrm{kg} /$ Mcal, whereas the PMEAN( \pm PSD) of $\bar{\phi}_{(32)}^{e}$ was $0.07( \pm 0.04) \mathrm{kg} / \mathrm{kg}^{0.75}$ such that, for example, as the residual or temporary environment effect of MBW increases by $1 \mathrm{~kg}^{0.75}$, the residual effect of DMI is estimated to increase by $0.07 \mathrm{~kg}$ holding constant MILKE. These overall genetic and residual PE estimates are in reasonable agreement with averages of station specific partial regression coefficients relating DMI to MILKE and to MBW within a classical RFI analysis on the same data reported previously by Tempelman et al. (2015) who, in turn, demonstrated these estimates to be consistent with estimates reported by NRC (2001).

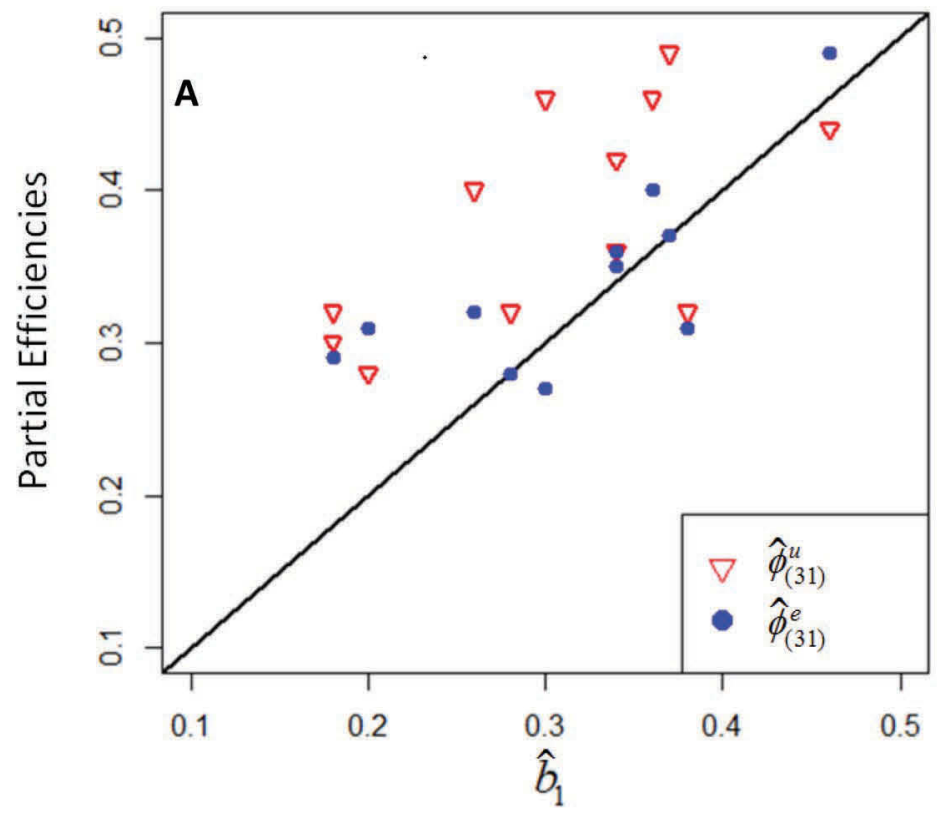

A scatterplot of the station-specific marginal means for genetic and residual $\mathrm{PE}$ versus the corresponding partial regression coefficients relating DMI to MILKE and to MBW from the classical RFI analyses in Tempelman et al. (2015) are provided in Figure 1, noting that estimates from station $\mathrm{AB}$ are not included in Figure 1 because that station was not considered in Tempelman et al. (2015). Correlations between station-specific partial regression coefficients relating DMI to MILKE, reported by Tempelman et al. (2015), with corresponding genetic and residual PE estimates were 0.66 and 0.76 , respectively. Correlations between station-specific partial regression coefficients relating DMI to MBW, reported by Tempelman et al. (2015), with corresponding genetic and residual PE estimates were 0.39 and 0.56 , respectively. Although the agreement between the 2 analyses was generally good, it should be clearly seen that the genetic PE were generally larger than residual PE, particularly for DMI on MILKE (Figure 1A). Furthermore, there appeared to be less heterogeneity between stations in the genetic $\mathrm{PE}$ relative to the residual PE for DMI on MBW (Figure 1B).

A list of these same station-specific marginal mean inferences for the genetic and residual $\mathrm{PE}$ are provided in Table 2 for DMI on MILKE and in Table 3 for DMI on MBW. Inference summaries are also provided for primiparous versus multiparous animals as well as for linear and quadratic coefficients of DIM on these PE. Although the estimated marginal mean genetic PE be-

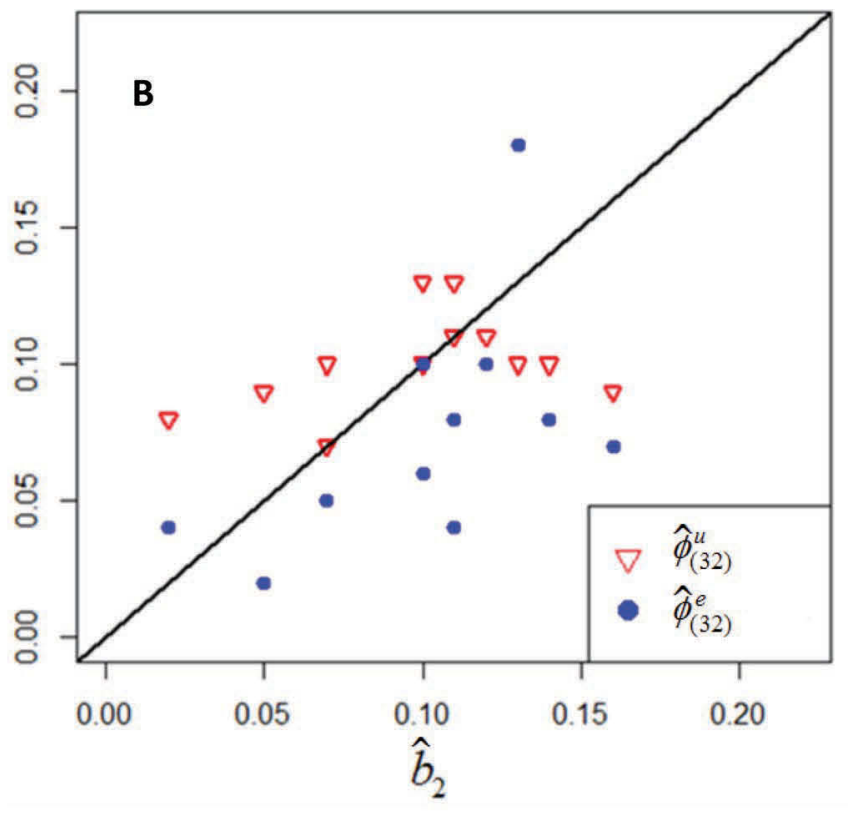

Figure 1. Posterior means of station-specific genetic $\left(\hat{\phi}_{(31)}^{u}\right.$ and $\left.\hat{\phi}_{(32)}^{u}\right)$ and residual $\left(\hat{\phi}_{(31)}^{e}\right.$ and $\left.\hat{\phi}_{(32)}^{e}\right)$ partial efficiencies versus estimated partial regression coefficients $\left(\hat{b}_{1}\right.$ and $\left.\hat{b}_{2}\right)$ previously reported by Tempelman et al. (2015) for DMI (kg) on milk energy (MILKE, Mcal; panel A) and metabolic BW (MBW, kg; panel B). Color version available online. 
tween DMI and MILKE ranged widely from 0.28 to $0.49 \mathrm{~kg} /$ Mcal across stations, there was no formal evidence of any difference $(P>0.05)$ between any pair of stations or between primiparous versus multiparous cows. Variation due to ration within station effects on genetic PE between DMI and MILKE seemed substantial, based on the reported point estimate $\sqrt{\hat{\sigma}_{m_{u}(31)}^{2}}=0.07$ $\mathrm{kg} /$ Mcal such that one might anticipate roughly a range of $4 \times(0.07)=0.28 \mathrm{~kg} /$ Mcal difference on genetic PE between the most extreme ration effects, assuming normality and adjusting for station and DIM effects. As with the genetic PE, any differences in the marginal mean residual PE of DMI to MILKE did not appear to be important even though again point estimates varied widely from 0.27 to $0.49 \mathrm{~kg} /$ Mcal. Furthermore, the estimate $\sqrt{\hat{\sigma}_{m_{e}(31)}^{2}}=0.06 \mathrm{~kg} /$ Mcal further suggested that ration within station could be a substantial source of variability for the residual $\mathrm{PE}$ relationship between DMI and MILKE. Linear and quadratic effects of DIM also did not seem to be important for either residual or genetic PE; in other words, stage of lactation did not appear to be important for modeling PE between DMI and MILKE.

The posterior summary of station and parity level effects on the genetic and residual PE of DMI to MBW are provided in Table 3. There appeared to be no significant differences between stations for the genetic $\mathrm{PE}$ although point estimates ranged from 0.07 to $0.21 \mathrm{~kg} /$ $\mathrm{kg}^{0.75}$. Furthermore, heterogeneity in genetic PE of DMI to MBW due to the rations appeared to be relatively substantial $\left(\sqrt{\hat{\sigma}_{m_{u}(32)}^{2}}=0.024 \mathrm{~kg} / \mathrm{kg}^{0.75}\right)$. However, at the residual level, there was formal evidence of at least one difference for PE of DMI on MBW between ZOM $(0.05$ $\left.\pm 0.02 \mathrm{~kg} / \mathrm{kg}^{0.75}\right)$ and USDFRC $\left(0.13 \pm 0.04 \mathrm{~kg} / \mathrm{kg}^{0.75}\right)$ even though point estimates \pm standard errors ranged more widely from $0.02 \pm 0.04 \mathrm{~kg} / \mathrm{kg}^{0.75}$ (SAC) to 0.18 $\pm 0.12 \mathrm{~kg} / \mathrm{kg}^{0.75}$ (UF). The magnitude of variation in the residual PE of DMI on MBW due to the ration was again rather substantial $\left(\sqrt{\hat{\sigma}_{m_{e}(32)}^{2}}=0.026 \mathrm{~kg} / \mathrm{kg}^{0.75}\right)$.

\section{Inferences on Variance Components and Heritability of FE}

Supplemental Tables S2 and S3 (https://doi. org/10.3168/jds.2016-11491), and Table 4 summarize marginal inferences on fixed and random effects influencing VC for MILKE, MBW|MILKE, and DMI|MILKE,MBW, respectively. We focus in particular on DMI|MILKE,MBW (i.e., our proposed FE trait). The overall genetic variance as based on the PMEAN $( \pm \mathrm{PSD})$ of

$$
\bar{\sigma}_{u 3 \mid 1,2}^{2}=\frac{1}{n} \sum_{i=1}^{n} \exp \left(\mathbf{x}_{i}^{u \prime} \log \left(\hat{\boldsymbol{\tau}}_{(3 \mid, 2)}^{u}\right)+\mathbf{z}_{i}^{u \prime} \log \left(\hat{\mathbf{v}}_{(3 \mid 1,2)}^{u}\right)\right)
$$

was $0.65( \pm 0.19) \mathrm{kg}^{2}$, whereas the overall residual variance was based on the PMEAN ( \pm PSD) of

$$
\overline{\mathbf{\sigma}}_{e(3 \mid 1,2)}^{2}=\frac{1}{n} \sum_{i=1}^{n} \exp \left(\mathbf{x}_{i}^{e_{1}} \log \left(\hat{\boldsymbol{\tau}}_{3 \mid 1,2}^{e}\right)+\mathbf{z}_{i}^{e_{1}} \log \left(\hat{\mathbf{v}}_{3 \mid, 2}^{e}\right)\right)
$$

being 1.86( \pm 0.45$) \mathrm{kg}^{2}$, such that the PMEAN $( \pm$ PSD) of overall heritability was $0.26( \pm 0.08)$. Evidence for heterogeneity in the genetic variance for $\mathrm{FE}$ was considerable (Table 4). The NBZ station appeared to have the smallest estimated genetic variance $(0.47 \pm 0.10$ $\mathrm{kg}^{2}$ ) whereas the largest genetic variance was estimated at UF $\left(1.28 \pm 0.41 \mathrm{~kg}^{2}\right)$ among all stations. No significant differences in genetic variance in $\mathrm{FE}$ were found between primiparous and multiparous cows. Furthermore, genetic variances were demonstrated to be highly heterogeneous between rations within stations with the 95\% HPD for the CV on genetic variance between rations being between 0.26 and 0.52 .

The magnitude of heterogeneity on the residual variance of $\mathrm{FE}$ across research stations was also quite considerable (Table 4). The marginal PMEAN ( \pm PSD) for stations ranged from a low of $0.85( \pm 0.24) \mathrm{kg}^{2}$ at NLN to $3.01( \pm 1.25) \mathrm{kg}^{2}$ at LAN. Furthermore, primiparous cows were inferred to have a substantially lower residual variance $\left(1.01 \pm 0.18 \mathrm{~kg}^{2}\right)$ compared with multiparous cows $\left(2.91 \pm 0.47 \mathrm{~kg}^{2}\right)$. Also, there appeared to be considerable evidence for heterogeneity of residual variances across rations as the $95 \%$ HPD the $\mathrm{CV}$ on genetic variance between rations was defined as between 0.47 to 1.57 .

Table 5 summarizes inferences on heritabilities of FE across different stations and parity classes. Evidence in favor of heterogeneity of heritability was substantial with the smallest heritability estimated at LAN $(0.16 \pm$ $0.06)$ at the largest estimates at UF $(0.46 \pm 0.13)$ and NLN (0.41 \pm 0.09$)$. Furthermore, the estimated heritability $(0.39 \pm 0.06)$ for primiparous cows was almost twice that $(0.22 \pm 0.04)$ for multiparous cows with the difference being significant $(P<0.05)$.

Cross-Validation. The accuracies for the 5-fold cross-validation on the dairy consortium data are provided in Table 6. There was strong evidence for differences in model fit between the homogeneous $\left(\mathrm{M}_{0}\right)$ and fully heterogeneous $\left(\mathrm{M}_{4}\right)$ models. The accuracy for predicting DMI was consistently higher $(\sim 4 \%)$ using the heterogeneous model compared with that of the homogeneous model, which further implies that substantial heterogeneity of some nature (i.e., on genetic 
Table 4. Posterior inferences characterizing heterogeneity of genetic and residual variation of DMI (kg) conditional on milk energy (Mcal) and metabolic BW $(\mathrm{kg})$

\begin{tabular}{|c|c|c|c|c|c|c|c|}
\hline \multirow[b]{2}{*}{ Factor } & \multirow[b]{2}{*}{ Level } & $\operatorname{PMEAN}(\mathrm{PSD})^{1}$ & $95 \% \mathrm{HPD}^{2}$ & $\mathrm{ESS}^{3}$ & PMEAN (PSD) $)^{1}$ & $95 \% \mathrm{HPD}^{2}$ & $\mathrm{ESS}^{3}$ \\
\hline & & \multicolumn{3}{|c|}{ Genetic variance $\left(\mathrm{Mcal}^{4}\right)$} & \multicolumn{3}{|c|}{ Residual variance $\left(\mathrm{Mcal}^{4}\right)$} \\
\hline \multirow[t]{13}{*}{ Station $^{5}$} & $\mathrm{AB}$ & $1.18^{\mathrm{ab}}(0.46)$ & {$[0.44,2.09]$} & 620 & $2.41^{\mathrm{a}}(1.11)$ & {$[0.69,4.60]$} & 579 \\
\hline & BARC & $0.68^{\text {abcd }}(0.23)$ & {$[0.32,1.14]$} & 1,846 & $2.17^{\mathrm{ab}}(1.06)$ & {$[0.71,4.30]$} & 920 \\
\hline & ISU & $0.81^{\text {abcd }}(0.21)$ & {$[0.43,1.23]$} & 865 & $2.15^{\mathrm{a}}(0.89)$ & {$[0.86,3.94]$} & 412 \\
\hline & MSU & $0.69^{\text {abcd }}(0.18)$ & {$[0.37,1.05]$} & 985 & $1.32^{\mathrm{ab}}(0.46)$ & {$[0.55,2.21]$} & 904 \\
\hline & UF & $1.24^{\mathrm{ab}}(0.41)$ & {$[0.52,2.04]$} & 693 & $1.49^{\mathrm{ab}}(0.62)$ & {$[0.55,2.71]$} & 1,047 \\
\hline & USDFRC & $0.98^{\mathrm{b}}(0.20)$ & {$[0.60,1.38]$} & 630 & $1.88^{\mathrm{a}}(0.57)$ & {$[0.86,3.01]$} & 734 \\
\hline & UW & $0.77^{\text {abcd }}(0.20)$ & {$[0.41,1.17]$} & 663 & $1.38^{\mathrm{ab}}(0.45)$ & {$[0.61,2.27]$} & 792 \\
\hline & NBZ & $0.47^{\mathrm{c}}(0.10)$ & {$[0.28,0.67]$} & 260 & $1.56^{\mathrm{ab}}(0.66)$ & {$[0.53,2.84]$} & 1,431 \\
\hline & NLN & $0.57^{\text {acd }}(0.12)$ & {$[0.34,0.80]$} & 711 & $0.85^{\mathrm{b}}(0.24)$ & {$[0.43,1.34]$} & 686 \\
\hline & TGEN & $0.61^{\mathrm{abcd}}(0.20)$ & {$[0.29,1.00]$} & 968 & $2.28^{\mathrm{ab}}(1.15)$ & {$[0.67,4.59]$} & 418 \\
\hline & ZOM & $0.76^{\mathrm{abd}}(0.10)$ & {$[0.56,0.96]$} & 312 & $1.7^{\mathrm{a}}(0.29)$ & {$[1.19,2.26]$} & 231 \\
\hline & LAN & $0.51^{\mathrm{cd}}(0.13)$ & {$[0.28,0.78]$} & 1,423 & $3.01^{\mathrm{a}}(1.25)$ & {$[1.08,5.38]$} & 380 \\
\hline & $\mathrm{SAC}$ & $0.78^{\text {abcd }}(0.23)$ & {$[0.39,1.26]$} & 1,448 & $2.58^{\mathrm{a}}(1.20)$ & {$[0.79,4.95]$} & 622 \\
\hline \multirow[t]{2}{*}{ Parity } & Primiparous & $0.63(0.07)$ & {$[0.48,0.78]$} & 438 & $1.01^{\mathrm{x}}(0.18)$ & {$[0.67,1.37]$} & 290 \\
\hline & Multiparous & $0.83(0.13)$ & {$[0.59,1.09]$} & 222 & $2.91^{\mathrm{y}}(0.47)$ & {$[2.06,3.86]$} & 286 \\
\hline \multirow[t]{3}{*}{ Ration (station) } & $\mathrm{CV}^{6}$ & & & & & & \\
\hline & Genetic & $0.37(0.08)$ & {$[0.26,0.52]$} & 600 & & & \\
\hline & Residual & & & & $0.89(0.56)$ & {$[0.47,1.57]$} & 2,542 \\
\hline
\end{tabular}

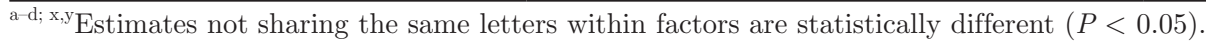

${ }^{1}$ Posterior (marginal) mean ( \pm posterior SD).

${ }^{2} 95 \%$ highest posterior density interval.

${ }^{3}$ Effective sample size.

${ }^{4}$ Milk energy (Mcal).

${ }^{5} \mathrm{AB}=$ University of Alberta; UF = University of Florida; ISU = Iowa State University; MSU = Michigan State University; UW = University of Wisconsin-Madison; USDFRC = USDA Dairy Forages Research Center; BARC = USDA Beltsville Agricultural Research Center; TGEN = 't Gen experimental herd, Lelystad, the Netherlands; NBZ = Nij Bosma Zathe, Leeuwarden, the Netherlands; ZOM = data based on the work of Zom et al. (2012); NLN = compilation of studies previously characterized by Tempelman et al. (2015); LAN = Langhill farm, Edinburgh, $\mathrm{UK} ; \mathrm{SAC}=$ Scottish Agricultural College.

${ }^{6}$ Coefficient of variation of ration within station-specific genetic and residual variance components.

Table 5. Inferences on station and parity specific heritabilities for DMI (kg) conditional on milk energy (Mcal) and metabolic BW $(\mathrm{kg})$

\begin{tabular}{llccr}
\hline Factor & Level & PMEAN $(\text { PSD })^{1}$ & $95 \%$ HPD $^{2}$ & ESS $^{3}$ \\
\hline Station $^{4}$ & AB & $0.35^{\mathrm{ab}}(0.14)$ & {$[0.10,0.62]$} & 668 \\
& BARC & $0.26^{\mathrm{ab}}(0.10)$ & {$[0.08,0.47]$} & 1,003 \\
& ISU & $0.29^{\mathrm{ab}}(0.10)$ & {$[0.12,0.50]$} & 539 \\
& MSU & $0.35^{\mathrm{ab}}(0.10)$ & {$[0.16,0.56]$} & 652 \\
& UF & $0.46^{\mathrm{a}}(0.13)$ & {$[0.18,0.55]$} & 922 \\
& USDFRC & $0.36^{\mathrm{ab}}(0.10)$ & {$[0.18,0.57]$} & 460 \\
& UW & $0.37^{\mathrm{ab}}(0.10)$ & {$[0.09,0.42]$} & 701 \\
& NBZ & $0.25^{\mathrm{ab}}(0.09)$ & {$[0.22,0.57]$} & 670 \\
& NLN & $0.41^{\mathrm{a}}(0.09)$ & {$[0.21,0.41]$} & 540 \\
TGEN & $0.24^{\mathrm{ab}}(0.10)$ & {$[0.05,0.29]$} & 254 \\
Parity & ZOM & $0.31^{\mathrm{a}}(0.05)$ & {$[0.08,0.45]$} & 667 \\
& LAN & $0.16^{\mathrm{b}}(0.06)$ & {$[0.27,0.50]$} & 804 \\
& SAC & $0.25^{\mathrm{ab}}(0.10)$ & {$[0.14,0.31]$} & 342 \\
\hline
\end{tabular}

a,b; x,y Estimates not sharing the same letters within factors are statistically different $(P<0.05)$.

${ }^{1}$ Posterior mean ( \pm posterior SD).

${ }^{2} 95 \%$ highest posterior density interval.

${ }^{3}$ Effective sample size.

${ }^{4} \mathrm{AB}=$ University of Alberta; UF = University of Florida; ISU = Iowa State University; MSU = Michigan State University; UW = University of Wisconsin-Madison; USDFRC = USDA Dairy Forages Research Center; $\mathrm{BARC}=\mathrm{USDA}$ Beltsville Agricultural Research Center; TGEN = 't Gen experimental herd, Lelystad, the Netherlands; NBZ = Nij Bosma Zathe, Leeuwarden, the Netherlands; ZOM = data based on the work of Zom et al. (2012); NLN = compilation of studies previously characterized by Tempelman et al. (2015); LAN = Langhill farm, Edinburgh, UK; SAC = Scottish Agricultural College. 
Table 6. Cross-validation prediction accuracies for each of 5 different validation data sets (fold) for homogeneous and heterogeneous models

\begin{tabular}{lcc}
\hline \multirow{2}{*}{ Fold } & \multicolumn{2}{c}{ Combined } \\
\cline { 2 - 3 } & Homogeneous $\left(\mathrm{M}_{0}\right)$ & Heterogeneous $\left(\mathrm{M}_{4}\right)$ \\
\hline 1 & 0.75 & 0.78 \\
2 & 0.78 & 0.82 \\
3 & 0.80 & 0.84 \\
4 & 0.77 & 0.80 \\
5 & 0.82 & 0.85 \\
Mean & 0.78 & 0.82 \\
\hline
\end{tabular}

or residual $\mathrm{PE}$, or on genetic or residual $\mathrm{VC}$ ) exists in this consortium data set.

Simulation Study Assessment. Coverage frequencies, ranging from $16 / 20$ to $20 / 20$ for the $95 \%$ HPD of key hyperparameters across the 20 replicates in the simulation study, are listed in Table 7 and were as nearly expected (i.e., 95\%). Summing across the last column of Table 7 , the overall coverage frequency across all parameters was $458 / 470$ or very close to the expected $95 \%$. The posterior means of $\gamma_{(r)}^{u}$ were more variable and their $95 \%$ HPD thus was wider than those of $\gamma_{(r)}^{e}$, as there is typically greater uncertainty for parameters characterizing distribution of random effects as opposed to those for residuals, as reported in an earlier study (Bello et al., 2010).

\section{DISCUSSION}

We have previously proposed a MT model analysis involving MILKE, MBW, and DMI using the Cholesky decomposition to provide a statistically more elegant approach for characterizing the genetic merit and heritability of FE relative to classical RFI approaches

Table 7. Summary on $95 \%$ highest posterior density interval (HPD) and coverage probabilities across 20 replicated data sets in simulation study

\begin{tabular}{|c|c|c|c|c|c|}
\hline Item $^{1}$ & & True value & $\begin{array}{l}\text { Range of } 95 \% \\
\text { HPD lower limits }\end{array}$ & $\begin{array}{l}\text { Range of } 95 \% \\
\text { HPD upper limits }\end{array}$ & $\begin{array}{c}\text { Coverage } \\
\text { probability }\end{array}$ \\
\hline \multirow[t]{6}{*}{ Genetic PE } & $\gamma_{(21)}^{u}$ & 0.35 & {$[-0.236,-0.182]$} & {$[0.298,0.369]$} & $17 / 20$ \\
\hline & $\gamma_{(31)}^{u}$ & 0.40 & {$[-0.019,0.028]$} & {$[0.419,0.455]$} & $20 / 20$ \\
\hline & $\gamma_{(32)}^{u}$ & 0.20 & {$[-0.034,-0.018]$} & {$[0.188,0.286]$} & $19 / 20$ \\
\hline & $\sigma_{m_{u}(21)}^{2}$ & 0.10 & {$[0.068,0.072]$} & {$[0.143,0.148]$} & $20 / 20$ \\
\hline & $\sigma_{m_{u}(31)}^{2}$ & 0.10 & {$[0.067,0.068]$} & {$[0.130,0.133]$} & $20 / 20$ \\
\hline & $\sigma_{m_{u}(32)}^{2}$ & 0.10 & {$[0.065,0.101]$} & {$[0.124,0.129]$} & $19 / 20$ \\
\hline \multirow[t]{6}{*}{ Genetic VC } & $\tau_{(1)}^{u}$ & 4 & {$[1.288,1.637]$} & {$[3.932,4.302]$} & $17 / 20$ \\
\hline & $\tau_{(2 \mid 1)}^{u}$ & 8 & {$[6.413,6.753]$} & {$[12.537,13.632]$} & $20 / 20$ \\
\hline & $\tau_{(3 \mid 1,2)}^{u}$ & 1.5 & {$[0.859,1.948]$} & {$[2.056,2.496]$} & $18 / 20$ \\
\hline & $\eta_{(1)}^{u}$ & 8 & {$[2.002,8.046]$} & {$[21.108,27.842]$} & $19 / 20$ \\
\hline & $\eta_{(2 \mid 1)}^{u}$ & 8 & {$[3.268,4.693]$} & {$[12.525,15.652]$} & $20 / 20$ \\
\hline & $\eta_{(3 \mid 1,2)}^{u}$ & 8 & {$[2.000,2.962]$} & {$[15.473,24.727]$} & $20 / 20$ \\
\hline \multirow[t]{6}{*}{ Residual PE } & $\gamma_{21}^{e}$ & 0.30 & {$[0.043,0.084]$} & {$[0.430,0.472]$} & $20 / 20$ \\
\hline & $\gamma_{31}^{e}$ & 0.40 & {$[0.176,0.195]$} & {$[0.399,0.494]$} & $19 / 20$ \\
\hline & $\gamma_{32}^{e}$ & 0.30 & {$[0.174,0.192]$} & {$[0.402,0.425]$} & $20 / 20$ \\
\hline & $\sigma_{m_{e}(21)}^{2}$ & 0.10 & {$[0.068,0.071]$} & {$[0.140,0.144]$} & $20 / 20$ \\
\hline & $\sigma_{m_{e}(31)}^{2}$ & 0.10 & {$[0.084,0.109]$} & {$[0.197,0.202]$} & $18 / 20$ \\
\hline & $\sigma_{m_{e}(32)}^{2}$ & 0.10 & {$[0.071,0.073]$} & {$[0.133,0.137]$} & $20 / 20$ \\
\hline \multirow[t]{6}{*}{ Residual VC } & $\tau_{(1)}^{e}$ & 8 & {$[6.080,6.326]$} & {$[10.153,10.484]$} & $20 / 20$ \\
\hline & $\tau_{(2 \mid 1)}^{e}$ & 32 & {$[27.336,27.755]$} & {$[33.788,34.472]$} & $20 / 20$ \\
\hline & $\tau_{(3 \mid 1,2)}^{e}$ & 3 & {$[2.281,3.616]$} & {$[5.376,7.849]$} & $18 / 20$ \\
\hline & $\eta_{(1)}^{e}$ & 6 & {$[2.000,3.709]$} & {$[20.687,49.602]$} & $20 / 20$ \\
\hline & $\eta_{(2 \mid 1)}^{e}$ & 8 & {$[5.769,16.163]$} & {$[20.891,53.724]$} & $18 / 20$ \\
\hline & $\eta_{(3 \mid 1,2)}^{e}$ & 8 & {$[2.306,19.901]$} & {$[37.749,51.161]$} & $16 / 20$ \\
\hline
\end{tabular}

${ }^{1} \mathrm{PE}=$ partial efficiency; $\mathrm{VC}=$ variance component. 
(Lu et al., 2015). Note that the proposed MT model is more closely related than the classical specification of RFI to the genotypic regression model proposed by Kennedy et al. (1993), so the developments we have presented here somewhat represent a heterogeneous yet more elegant extension of their genotypic regression model. As we had also indicated in $\mathrm{Lu}$ et al. (2015), it certainly would have been ideal to include $\Delta \mathrm{BW}$ as a fourth trait given that, like MILKE and MBW, it is considered to be an important energy sink for lactating dairy cattle (Berry and Crowley, 2013). But, for reasons already outlined in $\mathrm{Lu}$ et al. (2015), we modeled it as a covariate to account for its effect on $\mathrm{FE}$ in our MT analyses. We further augment this work here by modeling heterogeneity in the genetic and residual (co) variances, albeit indirectly, between MILKE, MBW, and DMI, by specifying structural mixed effects models on each key parameter defined by the square root free Cholesky decomposition on the (co)variance matrices, similar to Bello et al. (2010). Based on formal model choice criteria (DIC), we determined that specifying such heterogeneity was important for the analysis of an international dairy feed efficiency consortium data set.

Due to computing constraints, we did not consider every possible submodel given that there are a total of 15 different structural model specifications, 3 per each of Equations [1], [5], [6], [7], and [8]. We might further conclude, nevertheless, that based on our analyses, specifying this heterogeneity was ultimately most important for $\mathrm{VC}$ and somewhat less so for PE, particularly for $\mathrm{PE}$ at the genetic level. The latter is not particularly too surprising because it is typically more challenging to detect heterogeneity in genetic (co)variances relative to residual (co)variances. It was somewhat surprising to us that we found no evidence of differences between parity classes for genetic or residual PE for DMI on MBW or for DMI on MILKE given that the metabolic relationships between these traits have been reported to differ between primiparous and multiparous cows (Wathes et al., 2007).

There was mild evidence of some heterogeneity in residual PE for DMI on MBW between stations with the only significant difference involving 2 research stations. Any differences in ration-specific or station-specific PE could be partly attributed to differences, for example, in management strategies, ration compositions, measurement error, or ambient temperature, for example, whether or not the animal is a thermo-neutral environment (NRC, 1981). Furthermore, we demonstrated that genetic PE were generally larger than residual PE, implying that the strength of the relationships between DMI with MILKE and MBW is stronger at the genetic level than at the residual level. In a classical RFI analysis, the residual and genetic $\mathrm{PE}$ are constrained to be identical as previously noted by Lu et al. (2015); nevertheless, estimates of these 2 sets of PE tracked very well with the corresponding partial regression coefficients from the station-specific RFI-based analyses conducted by Tempelman et al. (2015) as demonstrated earlier in Figure 1. Note that any heterogeneity in the PE, particularly at the genetic level, has implications for accurately estimating breeding values on $\mathrm{FE}$, because these PE determine the key relationship between the genetic merit of DMI and that of FE using Equation [3c], for example.

We concluded that the genetic PE of MBW|MILKE did not differ from 0 for any station, implying then no evidence of genetic association between MILKE and MBW. Our results are then consistent with near zero genetic correlation estimates between these 2 traits as reported elsewhere (Berry et al., 2003; Spurlock et al., 2012).

As previously indicated, there was much stronger evidence of a difference in genetic and residual variances and, consequently, in heritabilities for $\mathrm{FE}$ between research stations, adding further evidence that $\mathrm{FE}$ is indeed a complex trait as also indicated by Tempelman et al. (2015) who previously determined country-specific differences for heritabilities of RFI based on analyses of weekly data. The average heritability estimates reported in Table 6 appeared to be somewhat larger here than in Lu et al. (2015) and in Tempelman et al. (2015). Any differences between the current study with those 2 previous studies may be partly due to the fact that $42-\mathrm{d}$ data were used here instead of weekly data, such that the effect of $\triangle \mathrm{BW}$ was better characterized by relatively less measurement error in the current study. However, it could also reflect the fact that the modeled heterogeneity in genetic and residual PE in Equations [3c] and [4c] leads to more accurate characterizations of genetic and residual effects of FE (i.e., $u_{i(3 \mid 1,2)}$ and $e_{i(3 \mid 1,2)}$, respectively), thereby resulting in a more reliable estimates of the heritabilities of FE. Our analyses furthermore indicated that the heritability of FE in primiparous cows was nearly double that of multiparous cows due to the large difference between the parity classes for residual variance. Again, these results may partly reflect the differences in energy dynamics as it relates to growth demands in primiparous cows relative to multiparous cows. Because selection indices involving FE inherently depend upon the correct specification of genetic/residual variances and covariances between the key traits of MILKE, MBW, and DMI (Lu et al., 2015), it appears that differential selection index weightings may need to reflect this heterogeneity across stations and parities as well. It is also important to note that between-station heterogeneity may partly reflect differ- 
ences in time periods for data collection (see Table 1 in Tempelman et al., 2015), noting that higher heritability estimates tended to be associated with more recent data.

Although our genetic analysis is based on the use of pedigree information and not on genomic marker information to specific animal relationships, it is conceptually easy to extend the proposed multivariate model for genomic evaluations simply by replacing the $\mathbf{A}$ with the genomic or realized relationship matrix $\mathbf{G}$ or even a hybrid matrix $\mathbf{H}$ based on data from both genotyped and nongenotyped animals as with single step GBLUP (Aguilar et al., 2010). We focused on a pedigree-based analysis in this paper for one primary reason: $\mathbf{A}$ is a relatively sparse matrix with $83.6 \%$ of its entries being 0 , thereby allowing us to effectively use sparse matrix software (i.e., "SparseM" package in R) to help improve computational efficiency for sampling elements of $\mathbf{u}$, whereas $\mathbf{G}$ is typically a dense matrix. We also determined (not reported) inconsequential differences in EBV between using $\mathbf{A}$ versus $\mathbf{G}$ based on computationally tractable MT analyses assuming homogeneous PE and VC specifications per Lu et al. (2015). Nevertheless, we do believe it will be important to further optimize our software code to facilitate the incorporation of genomic information in future analyses.

One potential extension of our proposed model is to directly model genetic effects as random effects on genetic and residual PE and VC, much like what we did with ration effects. In other words, one could specify Equations [5], [6], [7], and [8] as a function of polygenic or even SNP-specific effects (i.e., in genomic) models. In fact, with respect to residual variances as per Equation [8], related work has already been conducted by Sorensen and Waagepetersen (2003) for polygenic effects and by Yang et al. (2011) for genomic marker effects. With respect to PE, somewhat indirectly related work has been conducted in beef cattle by Savietto et al. (2014) and in poultry (Aggrey and Rekaya, 2013; Rekaya and Aggrey, 2015) whereby animal specific random regressions are specified on partial regression relationships between DMI and MBW and ADG as based on a classical RFI model. At any rate, it seems likely that many more records would be needed than would be required here to infer upon genetic heterogeneity of this nature at these higher levels of the Bayesian model hierarchy. Our simulation study, nevertheless, indicates that it is possible to infer upon the nature of the heterogeneity specified in our proposed model.

\section{CONCLUSIONS}

We have conducted a hierarchical Bayesian analysis to infer upon potential heterogeneity on genetic and residual components of DMI conversion to MILKE and DMI to MBW as well as in genetic and residual VC of $\mathrm{FE}$ across research stations. We detected no evidence of heterogeneity in genetic PE with slight evidence of heterogeneity in the residual PE of DMI to MBW across research stations. However, evidence for genetic and residual heteroscedasticity was substantial across stations and rations within station levels for FE. The estimated heritability of $\mathrm{FE}$ ranged from 0.16 to 0.46 across stations, thereby implying that $\mathrm{FE}$ is a more complex trait than what is currently considered in most quantitative genetic analyses. Heterogeneous relationships across environments should be taken into consideration in the genetic characterization and management of $\mathrm{FE}$ in dairy cattle.

\section{ACKNOWLEDGMENTS}

The authors acknowledge funding from USDANational Institute of Food and Agriculture (Washington, DC; Grant Numbers 2011-68004-30340 and 2011-67015-30338). The technical support of George Wiggans (USDA Animal Genomics and Improvement Laboratory) in providing pedigree information for this study is also gratefully acknowledged.

\section{REFERENCES}

Aggrey, S. E., and R. Rekaya. 2013. Dissection of Koch's residual feed intake: Implications for selection. Poult. Sci. 92:2600-2605.

Aguilar, I., I. Misztal, D. L. Johnson, A. Legarra, S. Tsuruta, and T. J. Lawlor. 2010. Hot topic: A unified approach to utilize phenotypic, full pedigree, and genomic information for genetic evaluation of Holstein final score. J. Dairy Sci. 93:743-752.

Bello, N. M., J. P. Steibel, R. J. Erskine, and R. J. Tempelman. 2012. Inferring upon heterogeneous associations in dairy cattle performance using a bivariate hierarchical model. J. Agric. Biol. Environ. Stat. 17:142-161.

Bello, N. M., J. P. Steibel, and R. J. Tempelman. 2010. Hierarchical bayesian modeling of random and residual variance-covariance matrices in bivariate mixed effects models. Biom. J. 52:297-313.

Berry, D., and J. Pryce. 2014. Feed efficiency in growing and mature animals. In Proc. 10th World Congress on Genetics Applied to Livestock Production. ASAS, August 17-22, 2014, Vancouver, Canada.

Berry, D. P., F. Buckley, P. Dillon, R. D. Evans, M. Rath, and R. F. Veerkamp. 2003. Genetic relationships among body condition score, body weight, milk yield, and fertility in dairy cows. J. Dairy Sci. 86:2193-2204.

Berry, D. P., M. P. Coffey, J. E. Pryce, Y. de Haas, P. Lovendahl, N. Krattenmacher, J. J. Crowley, Z. Wang, D. Spurlock, K. Weigel, K. Macdonald, and R. F. Veerkamp. 2014. International genetic evaluations for feed intake in dairy cattle through the collation of data from multiple sources. J. Dairy Sci. 97:3894-3905.

Berry, D. P., and J. J. Crowley. 2013. Cell Biology Symposium: Genetics of feed efficiency in dairy and beef cattle. J. Anim. Sci. 91:1594-1613.

Brooks, S. P., and A. Gelman. 1998. General methods for monitoring convergence of iterative simulations. J. Comput. Graph. Stat. $7: 434-455$. 
Clayton, D. G. 1996. Generalized linear mixed models. Pages 275-301 in Markov Chain Monte Carlo in Practice. Springer, New York, NY.

Connor, E. E. 2015. Invited review: Improving feed efficiency in dairy production: Challenges and possibilities. Animal 9:395-408.

de Haas, Y., J. E. Pryce, M. P. Calus, E. Wall, D. P. Berry, P. Lovendahl, N. Krattenmacher, F. Miglior, K. Weigel, D. Spurlock, K. A. Macdonald, B. Hulsegge, and R. F. Veerkamp. 2015. Genomic prediction of dry matter intake in dairy cattle from an international data set consisting of research herds in Europe, North America, and Australasia. J. Dairy Sci. 98:6522-6534.

Gelman, A., J. Hill, and M. Yajima. 2012. Why we (usually) don't have to worry about multiple comparisons. J. Res. Educ. Eff. 5:189-211.

Kennedy, B. W., L. R. Schaeffer, and D. A. Sorensen. 1988. Genetic properties of animal models. J. Dairy Sci. 71:17-26.

Kennedy, B. W., J. H. J. Vanderwerf, and T. H. E. Meuwissen. 1993. Genetic and statistical properties of residual feed-intake. J. Anim. Sci. 71:3239-3250.

Kizilkaya, K., and R. J. Tempelman. 2005. A general approach to mixed effects modeling of residual variances in generalized linear mixed models. Genet. Sel. Evol. 37:31-56.

Littell, R. C., W. W. Stroup, R. J. Freund, and SAS Institute. 2002. SAS for linear models. 4th ed. SAS Institute Inc., Wiley, Cary, NC.

Lu, Y., M. J. Vandehaar, D. M. Spurlock, K. A. Weigel, L. E. Armentano, C. R. Staples, E. E. Connor, Z. Wang, N. M. Bello, and R. J. Tempelman. 2015. An alternative approach to modeling genetic merit of feed efficiency in dairy cattle. J. Dairy Sci. 98:6535-6551.

Manzanilla-Pech, C. I., R. F. Veerkamp, R. J. Tempelman, M. L. van Pelt, K. A. Weigel, M. VandeHaar, T. J. Lawlor, D. M. Spurlock, L. E. Armentano, C. R. Staples, M. Hanigan, and Y. De Haas. 2016. Genetic parameters between feed-intake-related traits and conformation in 2 separate dairy populations-The Netherlands and United States. J. Dairy Sci. 99:443-457.

Milliken, G. A., and D. E. Johnson. 2009. Analysis of Messy Data Volume 1: Designed Experiments. CRC Press, Boca Raton, FL.

NRC. 1981. Effect of environment on nutrient requirements of domestic animals. Natl. Acad. Press, Washington, DC.

NRC. 2001. Nutrient Requirements of Dairy Cattle. 7th rev. ed. Natl. Acad. Press, Washington, DC.
Plummer, M. N., K. C. Best, and K. Vines. 2006. CODA: Convergence diagnosis and output analysis for MCMC. Vol. 6. R News.

Pollak, E. J., J. Vanderwerf, and R. L. Quaas. 1984. Selection bias and multiple trait evaluation. J. Dairy Sci. 67:1590-1595.

Rekaya, R., and S. E. Aggrey. 2015. Genetic properties of residual feed intakes for maintenance and growth and the implications of error measurement. J. Anim. Sci. 93:944-948.

Savietto, D., D. P. Berry, and N. C. Friggens. 2014. Towards an improved estimation of the biological components of residual feed intake in growing cattle. J. Anim. Sci. 92:467-476.

Sorensen, D., and R. Waagepetersen. 2003. Normal linear models with genetically structured residual variance heterogeneity: A case study. Genet. Res. 82:207-222.

Spiegelhalter, D. J., N. G. Best, B. R. Carlin, and A. van der Linde. 2002. Bayesian measures of model complexity and fit. J. R. Stat. Soc. B 64:583-616.

Spurlock, D. M., J. C. Dekkers, R. Fernando, D. A. Koltes, and A. Wolc. 2012. Genetic parameters for energy balance, feed efficiency, and related traits in Holstein cattle. J. Dairy Sci. 95:5393-5402.

Tempelman, R. J., D. M. Spurlock, M. Coffey, R. F. Veerkamp, L. E. Armentano, K. A. Weigel, Y. de Haas, C. R. Staples, E. E. Connor, Y. Lu, and M. J. VandeHaar. 2015. Heterogeneity in genetic and nongenetic variation and energy sink relationships for residual feed intake across research stations and countries. J. Dairy Sci. 98:2013-2026.

Wathes, D. C., Z. Cheng, N. Bourne, V. J. Taylor, M. P. Coffey, and S. Brotherstone. 2007. Differences between primiparous and multiparous dairy cows in the inter-relationships between metabolic traits, milk yield and body condition score in the periparturient period. Domest. Anim. Endocrinol. 33:203-225.

Yang, Y., O. F. Christensen, and D. Sorensen. 2011. Use of genomic models to study genetic control of environmental variance. Genet. Res. (Camb.) 93:125-138.

Zom, R. L. G., G. Andre, and A. M. van Vuuren. 2012. Development of a model for the prediction of feed intake by dairy cows: 1 . Prediction of feed intake. Livest. Sci. 143:43-57. 